\title{
InGaN light-emitting diodes with indium-tin-oxide photonic crystal current-spreading layer
}

\author{
K. H. Li and H. W. Choi ${ }^{\text {a) }}$ \\ Department of Electrical and Electronic Engineering, The University of Hong Kong, Hong Kong
}

(Received 4 April 2011; accepted 31 July 2011; published online 8 September 2011)

\begin{abstract}
Photonic crystal patterns on the indium tin oxide layer of an InGaN/GaN light-emitting diode are fabricated via nanosphere lithography in combination with dry etching. The silica spheres acting as an etch mask are self-assembled into a hexagonal closed-packed monolayer array. After etching, the photonic crystal $(\mathrm{PhC})$ pattern is formed across the indium-tin-oxide (ITO) films so that the semiconductor layers are left intact and thus free of etch damages. Despite slight degradation to the electrical properties, the ITO-PhC light-emitting diodes (LEDs) exhibit enhancements of their optical emission power by as much as $64 \%$ over an as-grown LED. The optical performances and mechanisms of the photonic crystal LEDs are investigated with the aid of rigorous coupled wave analysis and finite-difference time-domain simulations. (C) 2011 American Institute of Physics. [doi:10.1063/1.3631797]
\end{abstract}

\section{INTRODUCTION}

In recent years, GaN-based light-emitting diodes (LEDs) have been widely used in a wide range of domestic, industrial, and scientific products such as desk lighting, panel display backlighting, and illuminations for microscopy, just to name a few. The growing demand for blue light LEDs has also prompted the development of devices with maximal external quantum efficiency, which is determined by both internal quantum efficiency (IQE) and light extraction efficiency (EQE). IQE has been greatly enhanced over the years due to massive improvements in crystal quality. IQEs exceeding 70\% have been demonstrated from metalorganic chemical vapor deposition (MOCVD) grown material on sapphire substrates. ${ }^{1}$ However, due to the large refractive contrast at the nitride-air interface, the majority of light emitted from the MQWs is totally internally reflected, resulting in low EQE. ${ }^{2}$ Numerous approaches have been adopted to efficiently extract light from devices and prevent unwanted guiding modes, such as geometrical shaping, ${ }^{3}$ micro-LEDs, ${ }^{4}$ and surface roughening. ${ }^{5}$ These methods rely on the formation of non-parallel surfaces to minimize reflections, albeit at different dimensional scales. Surface texturing techniques, however, involve plasma etching of the top $\mathrm{p}-\mathrm{GaN}$ contact layer, and the plasma damage induced will significantly degrade electrical conduction in the device due to increased Ohmic contact resistance and leakage currents, thus sacrificing the overall efficiency. Plasma damage of the $p-n$ junction has also been shown to affect the device lifetime. ${ }^{6}$ Instead of directly processing the GaN layer, surface roughening of the indium tin oxide (ITO) current spreading layer has been demonstrated as an alternative method for improving light extraction efficiency without degradation of the electrical characteristics. In a report by Kim et al., the light output powers of LEDs with a pillar patterned ITO contact layer by colloidal lithography were enhanced by $10 \% .^{7}$ In another

\footnotetext{
a) Author to whom correspondence should be addressed. Electronic mail: hwchoi@hku.hk. Fax: (852) 25598738.
}

report by the same authors, LEDs with a randomly textured ITO layer using a natural mask were found to effectively boost output powers via the scattering effect. ${ }^{8}$ For a more pronounced and systematic effect, photonic crystals (PhCs) may be formed onto the ITO layer instead. PhCs enable strong interaction of the guided modes with the periodic structure, offering better control over the directionality of light emission as compared to random surface texturing. Two major properties associated with $\mathrm{PhCs}$ are the photonic bandgap (PBG) for molding the flow of light and diffraction, both of which are useful for extracting the guided modes to different extents. As the characteristic length scales of $\mathrm{PhC}$ structures are on the order of the wavelength, nano-patterning techniques are involved during the fabrication of visible light devices, often increasing manufacturing costs. Apart from nano-patterning using e-beam ${ }^{9}$ or nano-imprint ${ }^{10}$ lithographic techniques, nanosphere lithography ${ }^{11}$ (NSL) has been demonstrated as a high-efficiency and low-cost nano-fabrication method. A selfassembled nanosphere array is perfectly capable of patterning the large-area periodic array required for a $\mathrm{PhC}$.

The fabrication and characterization of $\mathrm{PhC}$-on-ITO LEDs patterned via NSL are reported in this paper. The selfassembled array of spheres serves as a hard mask for pattern transfer onto the ITO layer, resulting in hexagonally closepacked ITO pillar arrays. Although such $\mathrm{PhC}$ structures do not possess a PBG in the visible region, light extraction can be improved via the dispersion effect. The guided modes are effectively diffracted by the periodic refractive lattice. The performance of the ITO-PhC LEDs is evaluated together with an investigation of the mechanisms involved. The rigorous coupled-wave analysis (RCWA) algorithm and finitedifference time-domain (FDTD) method are employed to investigate the effect of incorporating $\mathrm{PhCs}$ of different dimensions on the performance of LEDs.

\section{EXPERIMENTAL DETAILS}

A schematic diagram illustrating the process flow of ITO-PhC LEDs in this work is depicted in Fig. 1. The 
(a)

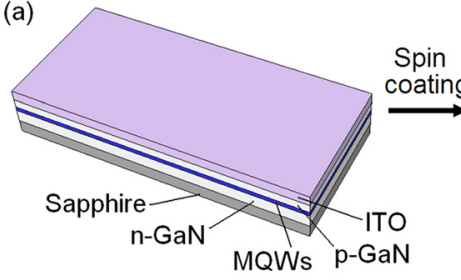

(d)
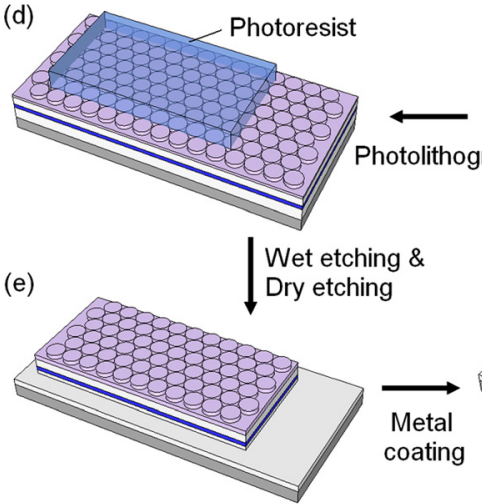

(b)

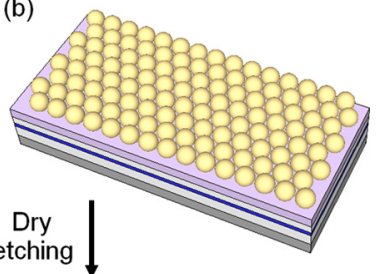

(c)
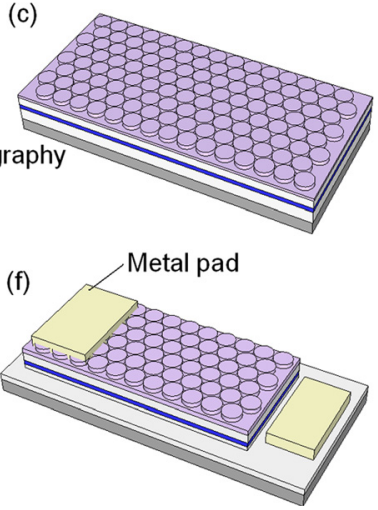

FIG. 1. (Color online) Schematic diagrams showing the process flow: (a) the starting wafer; (b) silica spheres spin-coated onto the ITO layer; (c) pattern transfer to the ITO layer via ICP etching followed by sphere removal; (d) mesa definition via photolithography; (e) exposure of the $\mathrm{n}-\mathrm{GaN}$ region after dry etching; and (f) metal pad deposition via e-beam evaporation.

InGaN/GaN LED wafers are grown on a c-plane sapphire substrate via MOCVD, with embedded multi-quantum wells designed for emission at around $470 \mathrm{~nm}$. A $200 \mathrm{~nm}$ thick transparent ITO coating is deposited via sputtering as a current spreading layer, as shown in Fig. 1(a). The ITO-PhC structure is patterned via NSL, beginning with the dispensing of a colloidal suspension onto the surface of the wafer using a micro-pipette. The colloidal suspension is prepared by mixing silica spheres with mean diameters of $500 \mathrm{~nm}, 700 \mathrm{~nm}$, and $1000 \mathrm{~nm}$ suspended in de-ionized water with an anionic surfactant sodium dodecyl sulfate. The spheres self-assemble naturally and uniformly across the ITO layer with the aid of spin-coating at optimized conditions. The rotation speed is varied between 140 and $200 \mathrm{rpm}$, depending on the sphere diameter, for a duration of $10 \mathrm{~min}$, resulting in the formation of a monolayer of spheres over an area of approximately $(8 \times 8) \mathrm{mm}^{2}$. The self-assembled hexagonal closed-packed array serves as an etch mask, the pattern of which is subsequently transferred to the ITO layer via inductively coupled plasma (ICP) etching. The etch parameters are set to $500 \mathrm{~W}$ of coil power and $150 \mathrm{~W}$ of platen power at $5 \mathrm{mTorr}$ of chamber pressure, using a gas chemistry comprising 15 SCCM of $\mathrm{Cl}_{2}$ and $10 \mathrm{SCCM}$ of Ar (SCCM denotes cubic centimeters per minute at standard temperature and pressure). Photolithographic patterning defines $600 \mu \mathrm{m}$ by 300 $\mu \mathrm{m}$ mesa regions, followed by dry etching to expose the n$\mathrm{GaN}$ layer. Another photolithographic step is performed to define the contact pad regions for metallization. The p-pads and $\mathrm{n}$-pads are deposited via e-beam evaporation, and the wafer is subjected to rapid thermal annealing at $500{ }^{\circ} \mathrm{C}$ in $\mathrm{N}_{2}$ ambient to form Ohmic contacts. For comparison, an unpatterned LED of identical dimensions is fabricated alongside it. The chips are diced by ultraviolet nanosecond laser micro-machining and die-bonded onto TO-headers, followed by $\mathrm{Al}$ wire-bonding. The surface morphologies of PhC LEDs are imaged by field-emission scanning electron microscopy (FE-SEM) using a Hitachi S-4800 system. The optical characteristics of the packaged LEDs are measured in an Ocean Optics 2 in. integrating sphere with a fiber-coupled radiometrically calibrated spectrometer (Ocean Optics HR-2000). The optical simulations are performed using RSoft software packages.

\section{RESULTS AND DISCUSSIONS}

Figure 2(a) shows an ordered hexagonal monolayer of nanosphere. In order to minimize the occurrence of defects such as dislocations and vacancies and avoid the formation of multiple layers, which disrupts the desired hexagonal pattern, the spin-coat rotation speed must be optimized. With decreasing sphere dimensions, an increase in the spin velocity (this centrifugal force) is required in order to overcome the viscosity of the suspension. The optimized speeds for achieving a high-quality monolayer array are determined to be 200,160 , and $140 \mathrm{rpm}$ for nanosphere diameters of 500 , 700, and $1000 \mathrm{~nm}$, respectively. After dry etching, periodic hexagonal-closed-packed ITO pillar arrays are formed with triangular air-gap voids between adjacent pillars exposed, as shown in Fig. 2(b). The etch depths of pillars are around 100 $\mathrm{nm}$, corresponding to an etching duration of $90 \mathrm{~s}$, as estimated from the SEM image in Fig. 2(c) captured at a tilted angle of $30^{\circ}$. Figures $2(\mathrm{~d})-2(\mathrm{f})$ show the resultant $\mathrm{PhC}$ structures after sphere removal with pillar diameters of 500, 700, and $1000 \mathrm{~nm}$, respectively.

Electroluminescence (EL) measurements are conducted on the packaged and un-encapsulated $\mathrm{PhC}$ and unpatterned

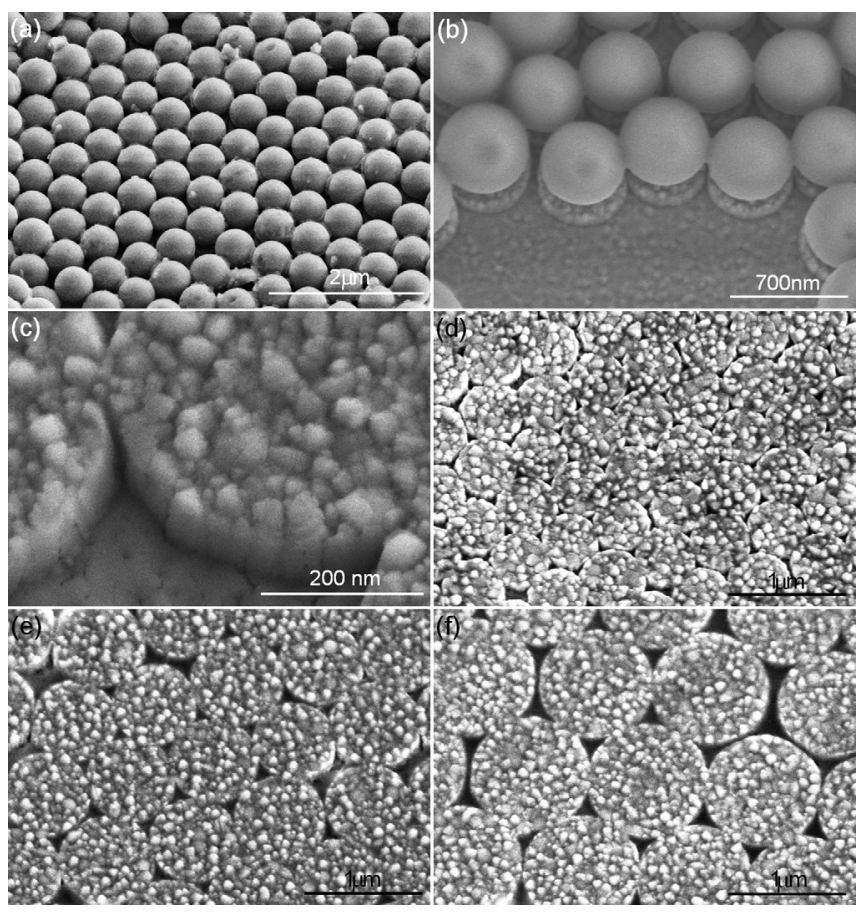

FIG. 2. FE-SEM images showing (a) ordered hexagonal monolayer arrays of nanospheres on a GaN LED wafer, (b) the nanosphere-coated sample after ICP etching, and (c) a close-up view of the ITO nano-pillars and the resultant nano-pillar arrays with diameters of (d) 500, (e) 700, and (f) $1000 \mathrm{~nm}$. 

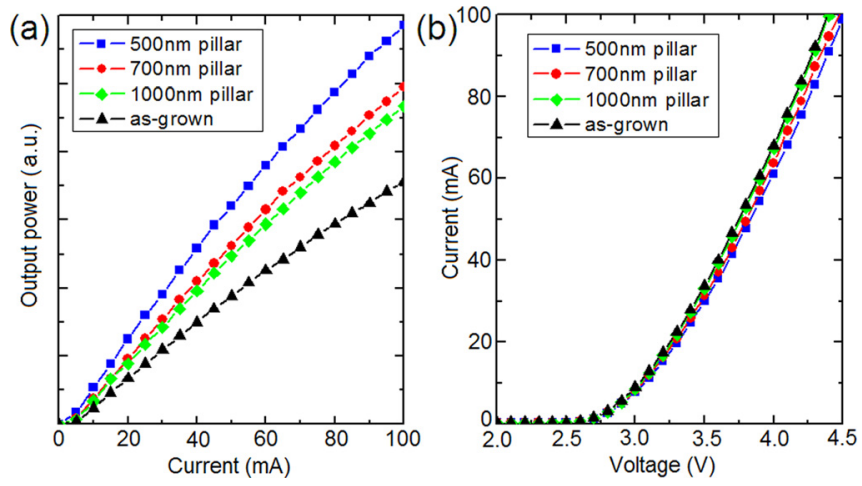

FIG. 3. (Color online) (a) Light output power as a function of injection currents and (b) I-V characteristics of PhC LEDs and as-grown LED samples.

LEDs by collecting the emitted light with a 2 in. integrating sphere optically coupled to a radiometrically calibrated spectrometer. Figure 3(a) shows plots of the EL intensity versus the injection current for the devices, from which it is observed that the PhC LEDs exhibit strong enhancements in light emission over the unpatterned LED. At an injection current of $100 \mathrm{~mA}$, the output powers of PhC LEDs with pillar diameters of 500,700 , and $1000 \mathrm{~nm}$ were enhanced by $64.6 \%, 39.1 \%$, and $31.2 \%$, respectively. The current-voltage (I-V) characteristics of the LEDs are plotted in Fig. 3(b). The forward voltages at $20 \mathrm{~mA}$ dc current are 3.30, 3.28, 3.26, and $3.25 \mathrm{~V}$ for PhC LEDs with diameters of 500, 700, and $1000 \mathrm{~nm}$ and the unpatterned LED, respectively. The slopes of the I-V curves in the linear region (thus series resistance) are also identical. The I-V data testify to the fact that nanostructuring of the ITO layer does not degrade the electrical characteristics of the LEDs, an important consideration for minimizing power consumption.

Figures 4(a)-4(d) show plan-view microphotographs of the $\mathrm{PhC}$ LEDs operated at $5 \mathrm{~mA}$ with pillar diameters of 500 , 700 , and $1000 \mathrm{~nm}$, together with the as-grown LED. As the ITO layer is not degraded by the micro-structuring, uniform emission is maintained. The PhC LEDs also appear brighter with decreasing pillar diameters. Compared with the $\mathrm{PhC}$ LEDs, the emission along the edges is significantly stronger than in the planar regions for the as-grown LED, because the guided photons either are reabsorbed within the active layer or escape through the sidewalls. The PhC LEDs, in contrast,

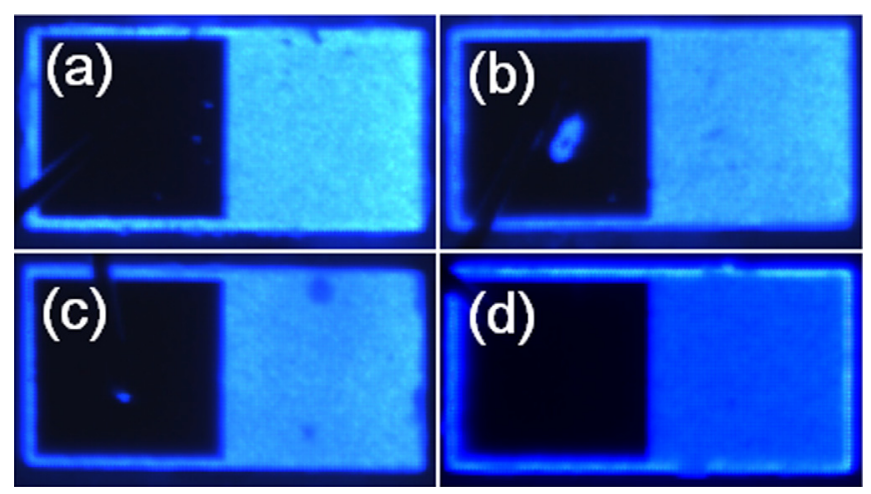

FIG. 4. (Color online) Optical microphotographs showing emission from ITO-PhC LEDs with pillar diameters of (a) $500 \mathrm{~nm}$, (b) $700 \mathrm{~nm}$, and (c) $1000 \mathrm{~nm}$ and (d) the as-grown LED. The devices are biased at $5 \mathrm{~mA}$.
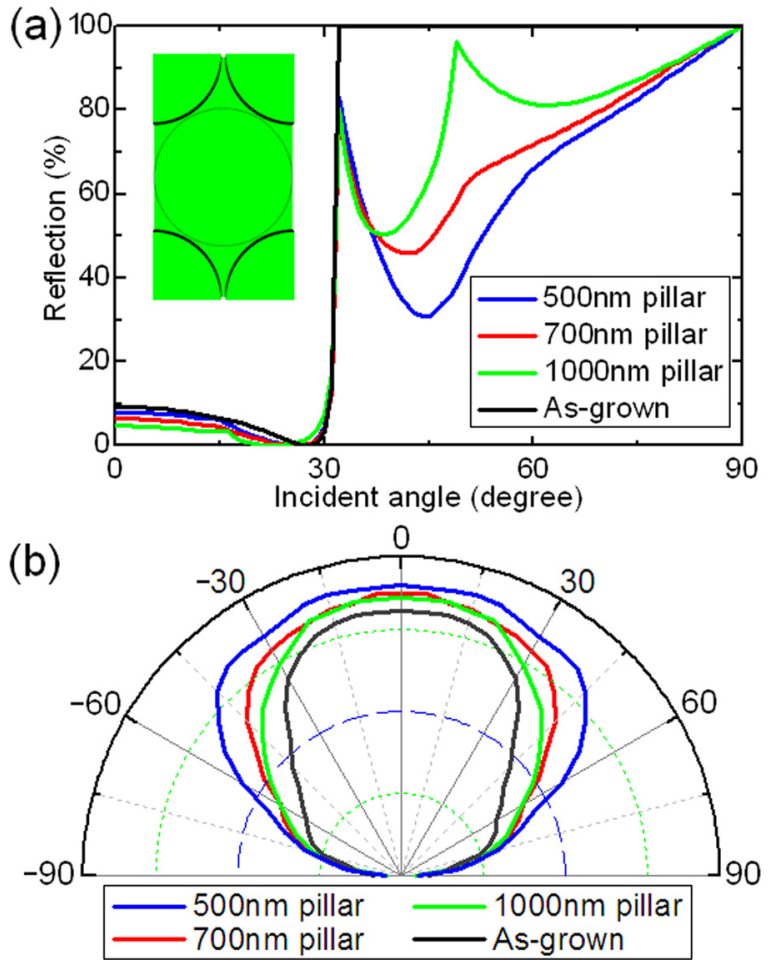

FIG. 5. (Color online) (a) Calculated reflection spectrum under varying incident angles. (b) Angular emission patterns of PhC LEDs and the as-grown sample.

offer an enhanced emission intensity over the entire planar surface. In order to investigate the function of the patterned ITO layer, a reflectivity simulation is performed based on the RCWA algorithm. The defined unit cell was as shown in the inset of Fig. 5(a), and a $\lambda=450 \mathrm{~nm}$ beam was incident onto the periodic array. At the ITO $\left(n_{\text {air }}=1.9\right)$-air $\left(n_{\text {ITO }}=1.0\right)$ interface, the critical angle determined by $\sin ^{-1}\left(n_{\text {air }} / n_{\text {ITO }}\right)$ is $\sim 31.8^{\circ}$, such that incident light rays striking the interface at angles greater than the critical angle are totally reflected, as illustrated in Fig. 5(a). In contrast, the ITO film incorporating PhCs serves as a light extraction layer for the suppression of total internal reflection, so that more photons are capable of escaping from the devices. Angular-resolved emission patterns of the LEDs are determined by collecting EL intensities with a fiber probe at different angles while maintaining a fiber-LED separation of $50 \mathrm{~mm}$. The light collected by the fiber is channeled to an optical spectrometer. The peak EL intensity at each angle is taken to plot the emission pattern, as shown in Fig. 5(b). For the as-grown LED, the intensity drops rapidly beyond $\sim 30^{\circ}$, and the full-width at half-maximum (FWHM). divergence angles increase with decreasing pillar diameters. The results are consistent with RCWA simulated predictions.

In order to further evaluate the effect of $\mathrm{PhC}$ patterns on LEDs, a three-dimensional FDTD simulation is carried out. Periodic boundary conditions are applied to the $\mathrm{x}-\mathrm{y}$ plane. The simulated LED structure consists of $200 \mathrm{~nm}$ thick ITO/150 nm thick p-GaN/40 nm thick MQWs/2000 nm GaN. The wavelength of the source is set as $450 \mathrm{~nm}$, and the mesh size is 20 $\mathrm{nm}$. A sufficient simulation period was allowed so that the light output signal attained steady-state. Figure 6(a) shows the 

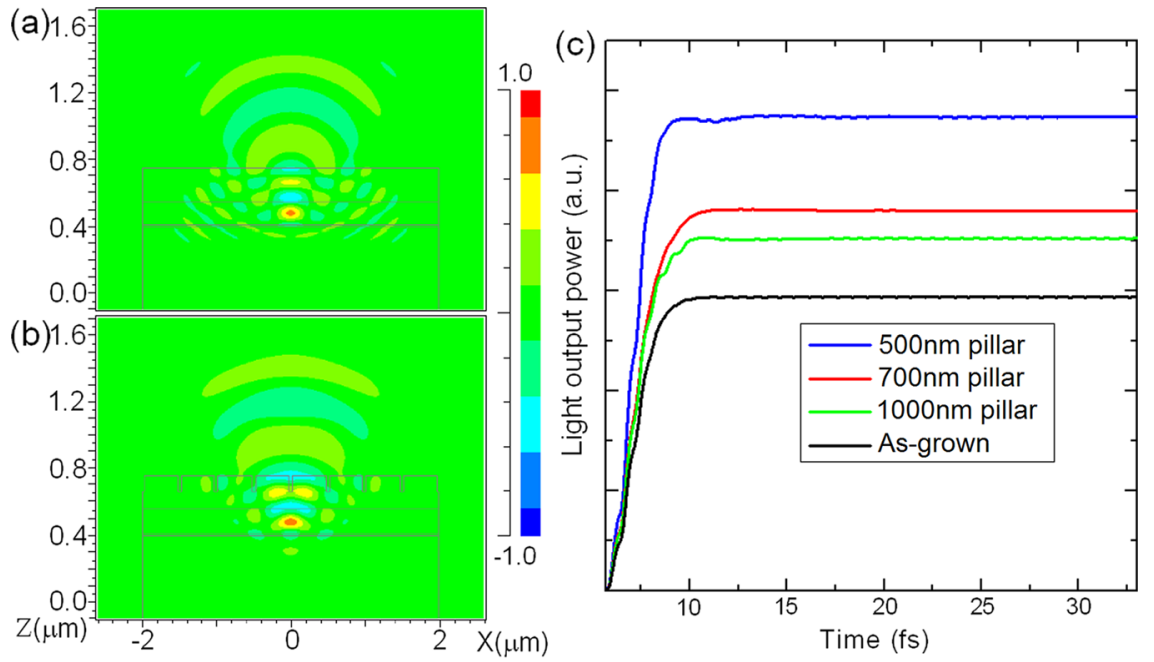

FIG. 6. (Color online) Comparison of FDTD simulations of the (a) as-grown flat-top and (b) $\mathrm{PhC}$ samples. (c) FDTD simulation results of the light output power of PhCs.

FDTD simulated emission pattern from an unpatterned LED; photons emitted outside the critical angle are seen to be totally reflected at the flat interface. In contrast, the incorporation of periodic PhC structures onto the ITO layer is seen to suppress lateral guiding modes and redirect the trapped photons into radiated modes, as illustrated in Fig. 6(b). The simulated timeresolved light intensity plot in Fig. 6(c) shows that PhC-LEDs with pillar diameters of 500, 700, and $1000 \mathrm{~nm}$ transmit $61.4 \%$, $29.5 \%$, and $20.0 \%$ more light than the flat-top sample, correlating well with previous experimental and simulated results.

FDTD simulations are also performed in order to study the effects of varying pillar heights on the optical output power. The heights of pillars are varied from $0 \mathrm{~nm}$ to 160 $\mathrm{nm}$ while the other device parameters remain unchanged. The computed output powers are normalized with respect to that of an un-patterned LED. From the simulated results plotted in Fig. 7, it is apparent that taller pillars generally deliver larger optical powers. It is also observed that the rate of increase of the output power for the $500 \mathrm{~nm} \mathrm{PhC} \mathrm{slows} \mathrm{down}$ significantly after the pillar exceeds a height of $\sim 100 \mathrm{~nm}$. As for the $700 \mathrm{~nm}$ and $1000 \mathrm{~nm} \mathrm{PhCs,} \mathrm{although} \mathrm{a} \mathrm{gradual}$ increase in the output power continues beyond pillar heights of $100 \mathrm{~nm}$, the higher degree of penetration into the ITO film would degrade the lateral conductivity of the current spread-

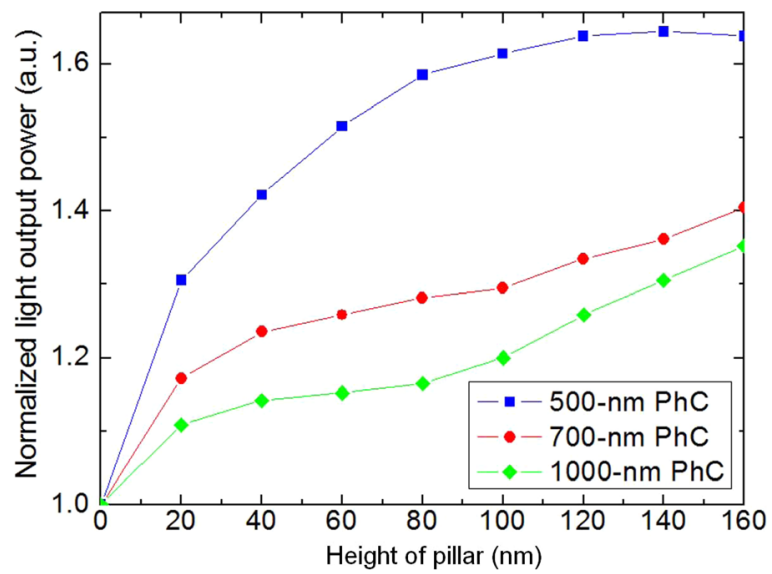

FIG. 7. (Color online) Plot of FDTD-computed normalized light output power as a function of pillar height for the 500, 700, and $1000 \mathrm{~nm} \mathrm{PhC}$ LEDs. ing layer and thus the electrical properties of device. In view of such considerations, the height of the pillar is designed to be $\sim 100 \mathrm{~nm}$, which is half of the total thickness of the ITO film, in order to maximize the overall device performance in terms of both optical and electrical characteristics.

Two-dimensional $\mathrm{PhCs}$ are known to promote light extraction in LEDs via two possible mechanisms. If the $\mathrm{PhC}$ possesses a PBG along the plane, the lateral guiding mode can effectively be eliminated over the range of frequencies covered by the bandgap. However, PhCs comprising closedpacked pillar structures as fabricated via NSL do not possess PBGs, as confirmed by the simulated TE and TM band diagrams shown in Fig. 8. Of course, it is possible to produce PBG structures using NSL. In our previous report, air-spaced nanopillar structures were fabricated by shrinking the patterned sphere pattern prior to pattern transfer. ${ }^{12}$ In this way, a PBG can be induced from such air-spaced pillar structures. In the present study, the "weak" PhCs serve to redirect emission from guided modes into radiative modes. ${ }^{13}$ A periodic refractive index is capable of altering the propagation behavior of a photon, as described by the dispersion relation $\omega(k)$ with the light line $\omega=k_{0} c$ for free-space propagation. According to Bloch's theorem, the dispersion curves of Bloch modes are folded at the Brillouin zone boundary, as is evident from Fig. 8. As a result, the waveguided modes originally located below the light line can be folded to the diffracted modes located above the light line, and thus can be extracted, provided the lattice constant is larger than the cutoff $\left(\Lambda_{\text {cutoff }}\right)$, which is evaluated by $\Lambda_{\text {cutoff }} \approx \lambda /\left(n_{\text {eff }}+1\right)$, where $n_{\text {eff }}$ is the effective index of the PhC layer. ${ }^{14}$ The lattice constants of the $\mathrm{PhC}$

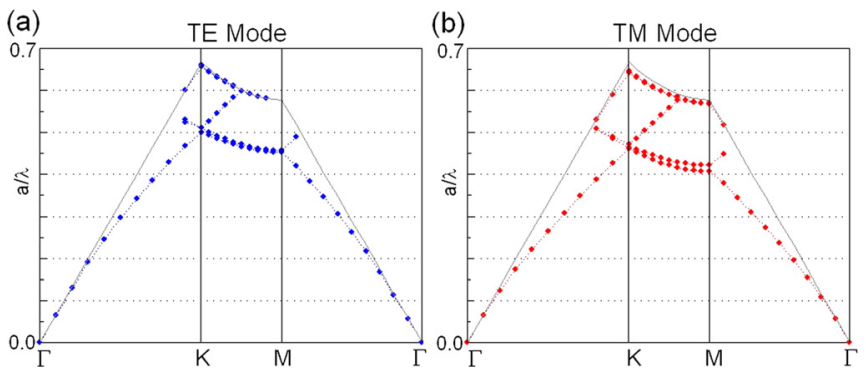

FIG. 8. (Color online) Simulated (a) TE and (b) TM band structures for closed-packed pillar arrays. 
LEDs described in this study are in the range of $500 \mathrm{~nm}$ to $1000 \mathrm{~nm}$, and thus satisfy the cutoff condition.

\section{CONCLUSION}

In summary, we have demonstrated the fabrication of LEDs with a PhC on the ITO current spreading layer, patterned via NSL. The self-assembled hexagonal-close-packed sphere array pattern is transferred to the ITO layer by dry etching. In this way, the semiconductor layers are not degraded in the process. No significant degradation to the ITO layer is observed, so that emission uniformity and good I-V characteristics are maintained. The emission powers increased with decreasing pillar diameters. In particular, the output power of the ITO-PhC LEDs with a diameter of $500 \mathrm{~nm}$ is enhanced by $64 \%$ compared to the un-pattern LED. The significant enhancement can be attributed to the dispersion behavior and diffraction property of PhCs. The measured results are verified by simulations based on the RCWA algorithm and the FDTD method.

\section{ACKNOWLEDGMENTS}

This work was supported by a GRF grant of the Research Grant Council of Hong Kong (project HKU 7118/09E).
${ }^{1}$ T. Akasaka, H. Gotoh, T. Saito, and T. Makimoto, Appl. Phys. Lett. 85, 3089 (2004).

${ }^{2}$ A. David, T. Fujii, R. Sharma, K. Mcgroody, S. Nakamura, S. P. DenBaars, E. L. Hu, and C. Weisbuch, Appl. Phys. Lett. 88, 061124 (2006).

${ }^{3}$ C. C. Kao, H. C. Kuo, H. W. Huang, J. T. Chu, Y. C. Peng, Y. L. Hsieh, C. Y. Luo, S. C. Wang, C. C. Yu, and C. F. Lin, IEEE Photon. Technol. Lett. 17, 19 (2005).

${ }^{4}$ H. W. Choi and S. J. Chua, J. Vac. Sci. Technol. B 24, 800 (2006).

${ }^{5}$ T. Fujii, Y. Gao, R. Sharma, E. L. Hu, S. P. DenBaars, and S. Nakamura, Appl. Phys. Lett. 84, 855 (2004).

${ }^{6}$ Z. L. Li, K. H. Li, and H. W. Choi, J. Appl. Phys. 108, 114511 (2010).

${ }^{7}$ T. K. Kim, S. M. Kim, Y. H. Jang, and G. Y. Jung, Appl. Phys. Lett. 91, 171114 (2007).

${ }^{8}$ T. K. Kim, S. H. Kim, S. S. Yang, J. K. Son, K. H. Lee, Y. G. Hong, K. H. Shim, J. W. Yang, K. Y. Lim, S. J. Bae, and G. M. Yang, Appl. Phys. Lett. 94, 161107 (2009).

${ }^{9}$ C. H. Lin, H. H. Yen, C. F. Lai, H. W. Huang, C. H. Chao, H. C. Kuo, T. C. Lu, S. C. Wang, and K. M. Leung, IEEE Photon. Technol. Lett. 20, 836 (2008).

${ }^{10}$ K. J. Byeon, S. Y. Hwang, and H. Lee, Appl. Phys. Lett. 91, 091106 (2007).

${ }^{11}$ J. C. Hulteen and R. P. Vanduyne, J. Vac. Sci. Technol. A 13, 1553 (1995).

${ }^{12}$ K. H. Li and H. W. Choi, J. Appl. Phys. 109, 023107 (2011).

${ }^{13}$ C. Wiesmann, K. Bergenek, N. Linder, and U. T. Schwarz, Laser Photonics Rev. 3, 262 (2009).

${ }^{14}$ Y. J. Lee, S. H. Kim, J. Huh, G. H. Kim, Y. H. Lee, S. H. Cho, Y. C. Kim, and Y. R. Do, Appl. Phys. Lett. 82, 3779 (2003). 